UDK 528.721.221.6

\title{
THE REGISTRATION OF POINT CLOUD DATA FROM RANGE IMAGING CAMERA
}

\author{
Cihan Altuntas ${ }^{1}$, Ferruh Yildiz ${ }^{2}$ \\ Department of Geomatics Engineering, Engineering Faculty, Selcuk University, 42075 Selcuklu, Konya, Turkey \\ E-mail: 1'caltuntas@selcuk.edu.tr (corresponding author)
}

Received 25 March 2013; accepted 18 September 2013

\begin{abstract}
The measurement and 3D modelling techniques have been evolved as parallel technological improvements. Every new technique provides an opportunity for low-cost and fast measurements. The latest method for 3D measurement is the range imaging (RIM) camera. The new period in photogrammetry and 3D modelling applications has begun with the RIM cameras, and it has brought new research areas for scientists. The measurement capabilities, accuracies and application areas of RIM cameras have been increased by the time. In this study, the registration of point cloud data of RIM camera was investigated to perform 3D modelling task.
\end{abstract}

Keywords: range imaging camera, time-of-flight camera, point cloud, registration, 3D modelling, photogrammetry.

Reference to this paper should be made as follows: Altuntas, C.; Yildiz, F. 2013. The registration of point cloud data from range imaging camera, Geodesy and Cartography 39(3): 106-112.

\section{Introduction}

The range imaging (RIM) camera is called with different names, i.e. range camera, laser range camera, 3DRIC camera, 3D depth camera, time-of-flight camera, CCD-D camera, and RGB-D camera. It acquires dense 3D point data instantly from the measurement site. It is a new method on spatial data measurement and 3D modelling, but their technical specifications and application areas have been increased rapidly by the time. So far, it has been used for many purposes, such as robotic, automotive, job safety, construction, deformation measurement etc.

The laser scanners cannot be used extensively in 3D modelling tasks due to high cost to own. In addition, because of its ground based measurement property, all object details could not be measured with it. However, RIM cameras can be moved everywhere to view object details. RIM cameras are attractive methods in many studies thanks to their instantly 3D measurement possibility. It can be used for 3D modelling studies as separately or together with the laser scanner and the other measurement techniques (Piatti 2010; Boehm, Pattinson 2010). The many measurements are performed by the RIM camera from different points of view to obtain occlusion free 3D model. The measurements can be performed either on static or kinematic (hand-held) modes. Each point cloud has its local coordinates center of which is the camera. Therefore, all successive measurements must be registered into reference coordinate system so as to complete 3D model. In this study, the measurements were practiced by RIM camera and their point clouds were registered into the reference coordinate system in order to get $3 \mathrm{D}$ object model.

\section{Background}

The RIM camera measures distance with time-of-flight principle of the laser light. The calibration and inspection of the camera sections have been conducted by Lichti et al. (2010) and Oggier et al. (2004). Especially, emitter, optics, electronic and sensor units had been carefully inspected, and they out performed that the camera can be used for 3D measurements similar to laser scanner. The distance measurement accuracy of SwissRanger camera was achieved in 5-10 millimeters, and carried out that the inside and outside 
temperatures have significantly affect on accuracy of RIM cameras at Kahlmann et al. (2006). The study has shown that the inside temperature is beginning to effect the measurements in a few minutes after the start of the camera, and it can be modelled as systematic error. The study was also demonstrated that the effect of outside temperature can be modelled likewise. Rapp (2007) investigated regular and irregular error sources and relationship between error and the range. In the study PMD (vision) 19k, SwissRanger SR-3000 and Effector O3D cameras had been inspected. It has shown that the measurements have systematic errors that can be modelled. In the study, the accuracy for SR3000 was acquired within $9 \mathrm{~mm}$, and this error was defined as acceptable value in 3D modelling study. In further study, the measurement precision of PMD CamCube 2.0 was examined for different lighting conditions and integration times (Centeno, Jutzi 2010).

Oggier et al. (2005) had provided automotive, interactive screen and biomedical applications of the camera. The position of the car according to location of ambient objects had determined by the distances that were measured by the camera. In the application for interactive screen, computer was controlled from projected screen by hand followed by the camera similar to touch screen. In addition, the face modelling was carried out in biological study. These applications can be performed with low coast and fast by the RIM camera in contrast to the photogrammetry and laser scanner. In another study, photogrammetric evaluation and the RIM measurement had been compared in that visualization, evaluation and convenience (Hussmann et al. 2008). According to the study, RIM camera had advantage in relation to driver aid, prevent of the crash, stop and go and detect of the pedestrian and bicycling on the road during car driving. At the study of Cui et al. (2010), the objects which have about $50 \mathrm{~cm}$ height had been modelled by the camera. The visualization defects of the measurements had been removed with repeated measurements. The camera can also be used to detection of moving. This attribute of the camera had been used to follow of the workers in construction and factory area in order to prevent accidents (Teizer 2008). Then early warning system has been set up to detect of the extraordinary moving, and the satisfying results had been achieved. Furthermore, hand-held mobile measurements can be achieved by the RIM camera. The camera had been used for distance measurement and route detection of the moving robots in Frank et al. (2010) and Sturm et al. (2010).
In this study, after the measurement principles of the RIM cameras were given, the measurements were practiced by the RIM camera and point clouds are registered into common coordinate system.

\section{Range imaging camera}

The RIM camera is instantly measure all points of the view unlike laser scanner. The measurements have their own local coordinates, the origin of that is the optic center of the camera. The camera records consecutive exposures which are about 50 frames per second (fps) (MesaImaging 2012). Each frame is recorded into a separate measurement file. When many frames are recorded from the same view, each one has little different value from the others because of many errors which are affect the measurements. Therefore, the mean measurement file is computed by the average of all consecutive exposures in order to reduce effect of the errors. According to Piatti (2010), thirty consecutive exposures ensure high accuracy 3D measurement by the RIM camera.

\section{Results}

Many measurements are performed by RIM camera from different point of view in order to view all details of the object which will be modelled. In this case, all point clouds that each one has local coordinates have to be registered into reference coordinate system. The point clouds of RIM camera can be registered by the iterative closest point (ICP) method (Besl, McKay 1992; Chen, Medioni 1992) similar to laser scanner tasks. The other way to perform the registration (rotations and translations) of point clouds is the use of amplitude or range images (Altuntas 2011). Three case studies were given below for explain the execution of these methods. The point clouds were registered by ICP in the first two case studies. In addition, handheld mobile measurement was performed by the camera at the third case study and the registrations were executed with the keypoints which were extracted by SIFT (Lowe 2004) from the amplitude images.

\subsection{Case study 1}

In this case, two overlapping measurements were performed by SR4000 camera. The camera is about 1.50 $\mathrm{m}$ and $1.60 \mathrm{~m}$ distance from the object for the first and second stations respectively (Fig. 1). The integration time was set to 3.3 milliseconds (ms), and 10, 30 and 50 consecutive frames were recorded from two stations (Fig. 2). 

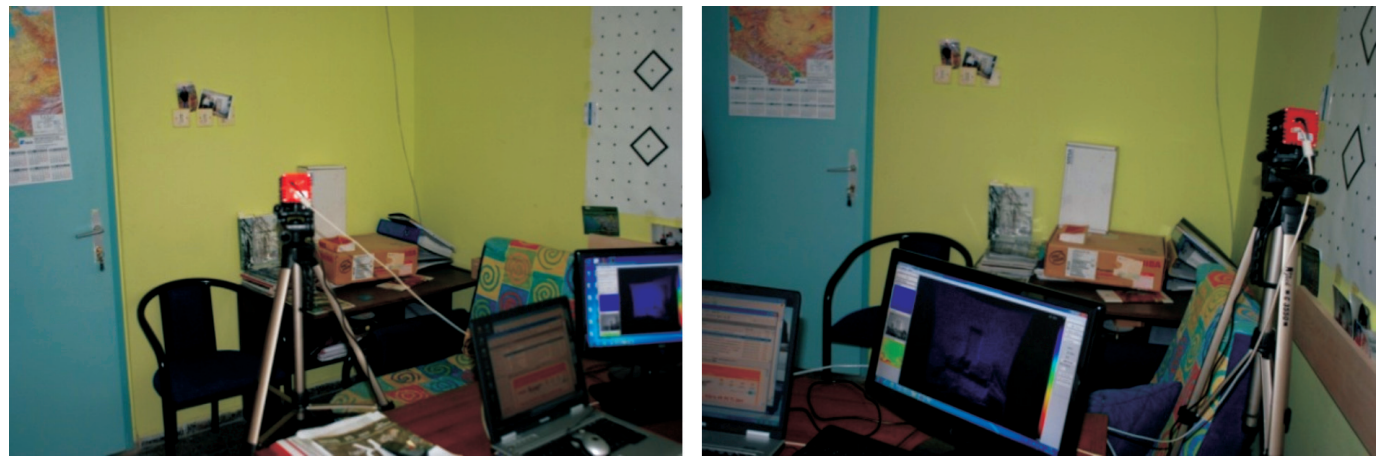

Fig. 1. SR4000 camera stations and measurement side (case study 1)
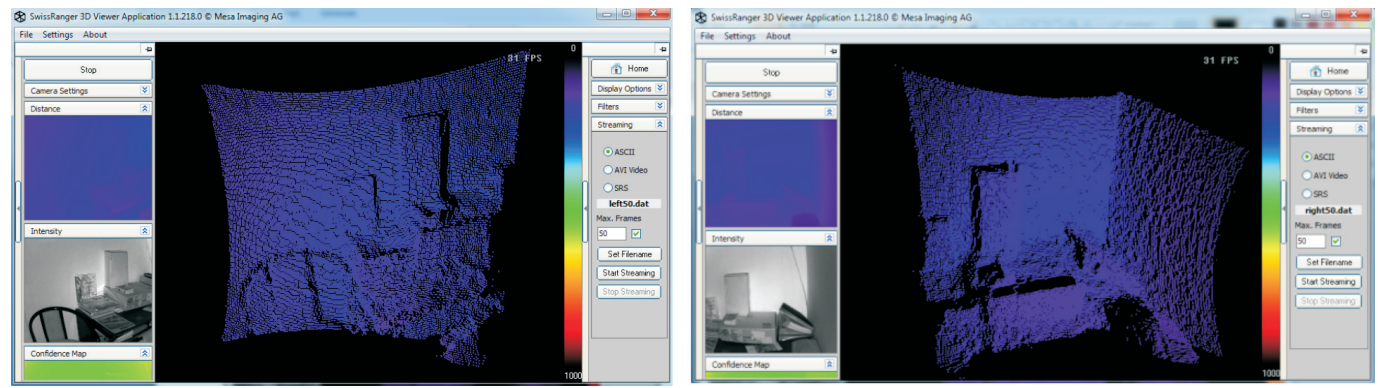

Fig. 2. The overlapping point clouds by SR4000 camera (case study 1)

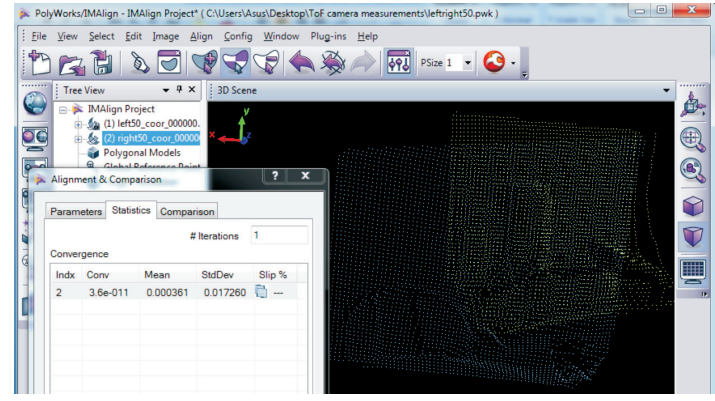

Fig. 3. The registered point clouds of RIM camera
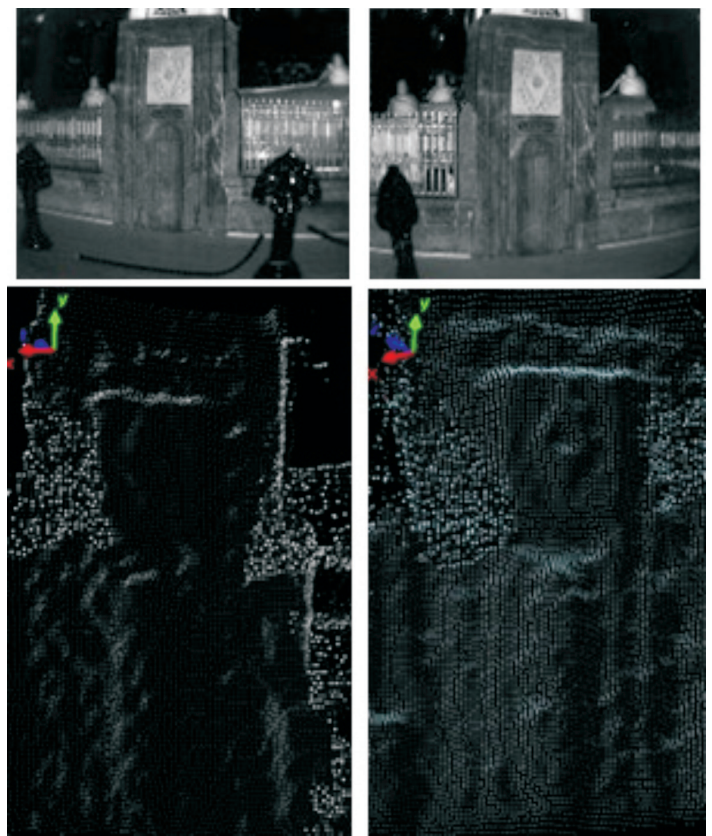

Fig. 4. The overlapping amplitude images (above) and point clouds (below) from two stations by SR4000 camera in Mevlana Museum
The mean measurement files of 10,30 and 50 consecutive frames were computed by created Matlab ${ }^{\circledast}$ code. Then the registration was realized between the same count measurements (Table 1) by using the ICP that was executed in PolyWorks ${ }^{\circledR}$ IMAlign (InnovMetric 2007) (Fig. 3).

\subsection{Case study 2}

The cultural heritage measurement was performed by the camera in the Mevlana Museum (Fig. 4). Once again 10,30, 50 consecutive frames were recorded from two stations with different point of view as overlapping. Then the mean measurement files were computed and the registration was realized between the same count measurements by the ICP (Table 1).

The Table 1 shows that the registration accuracies of the 30 and 50 consecutive frames are very close and it is slightly better than the 10 consecutive exposures. The number of the measurements affects accuracy of both the mean measurement and the registration. When the distances between the measured points, which are $0.6 \mathrm{~cm}$ and $2.09 \mathrm{~cm}$ (angular resolution is $0.24^{\circ}$ ) respectively in these two studies, are considered, the standard deviations are smaller than them. According to the results of these two studies, it can be said that thirty frames of consecutive exposure from the same view are enable high accuracy for 3D modelling studies, and the point clouds are registered with high accuracy by the ICP. Generally, ten frames also make it possible satisfying results in many studies. 
Table 1.The registration results of SR4000 point clouds by ICP

\begin{tabular}{|c|c|c|c|c|c|c|}
\hline \multirow{4}{*}{} & \multicolumn{2}{|c|}{ Station 1 } & \multicolumn{2}{c|}{ Station 2 } & \multicolumn{2}{c|}{ ICP registration } \\
\cline { 2 - 7 } & $\begin{array}{c}\text { Number } \\
\text { of frame }\end{array}$ & Distance $(\mathrm{m})$ & $\begin{array}{c}\text { Number } \\
\text { of frame }\end{array}$ & Distance $(\mathrm{m})$ & $\begin{array}{c}\text { Mean convergence } \\
(\mathrm{cm})\end{array}$ & $\begin{array}{c}\text { Std. dev. } \\
(\mathrm{cm})\end{array}$ \\
\hline \multirow{3}{*}{ Case study 1 } & 10 & 1.50 & 10 & 1.60 & 0.0414 & 1.6850 \\
\cline { 2 - 7 } & 30 & 1.50 & 30 & 1.60 & 0.0338 & 1.7221 \\
\cline { 2 - 7 } Case study 2 & 50 & 1.50 & 50 & 1.60 & 0.0361 & 1.7260 \\
\cline { 2 - 7 } & 10 & 4.90 & 10 & 5.00 & 0.0453 & 2.8305 \\
\cline { 2 - 7 } & 30 & 4.90 & 30 & 5.00 & 0.0331 & 3.0550 \\
\hline
\end{tabular}

\subsection{Case study 3}

The hand-held measurements need to solve some problems for the registration of point clouds. In such a situation, GPS/INS units have to be added to the camera so as to establish relationship (to solve three rotations and three translations) between the successive measurements as similar to aerial and ground based mobile LIDAR applications. However there is no GPS or INS with the camera. Therefore every frame is associated with the next successive images by point extraction or image matching methods (Piatti 2010). The registration parameters between the images are computed by the coordinates of the matched points. The SIFT (Lowe 2004) and SURF (Bay et al. 2008) are generally used for automatic image matching in simultaneous localization and mapping (SLAM) applications. The keypoints were extracted from the overlapping images and then matched by RANSAC (Fischler, Bolles 1981) in these methods (Brazetti et al. 2010; Altuntas 2011). The SIFT are widely used for automatic image matching with respect to the SURF.

The hand-held 100 consecutive frames were recorded by the SR4000 camera for the implementation of the method. During the measurement, the camera was moved through the side to view all details. Then the $1^{\text {st }}, 20^{\text {th }}, 40^{\text {th }}, 60^{\text {th }}, 80^{\text {th }}$ and $100^{\text {th }}$ frames (Fig. 5) were selected interactively from the measurements. The keypoints of the amplitude images of these frames were extracted by SIFT code given by Lowe (2005), and then matched. The outlier keypoints were removed by using fundamental (F) matrix as known in computer vision (Brazetti et al. 2010). Here, there are only three outlier keypoints between $80^{\text {th }}-100^{\text {th }}$ images, and they were removed from the matched point set.
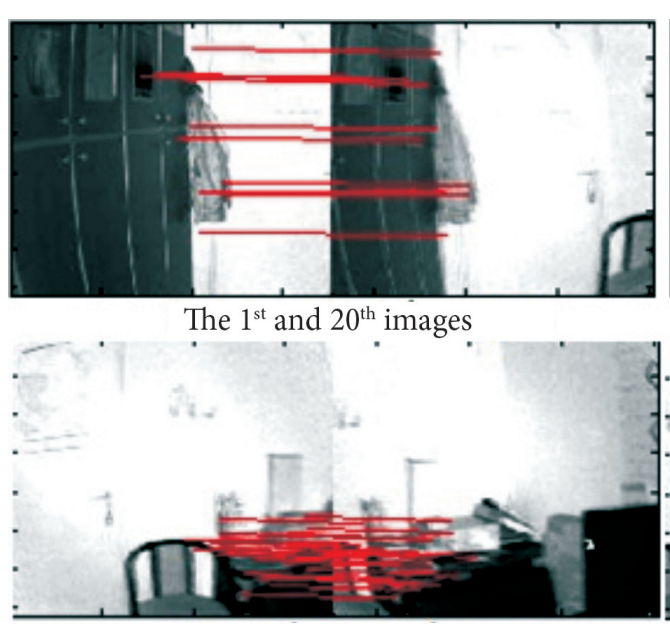

The $40^{\text {st }}$ and $60^{\text {th }}$ images

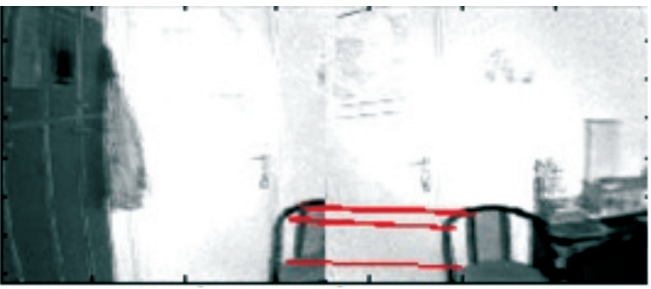

The $20^{\text {st }}$ and $40^{\text {th }}$ images

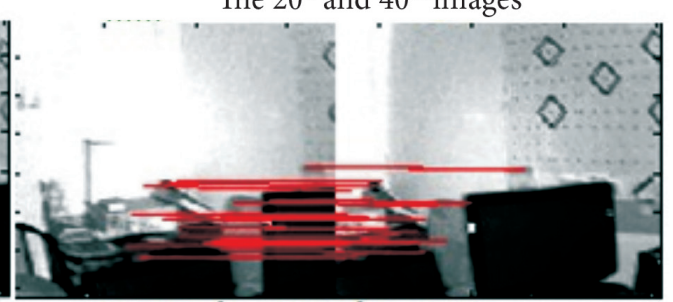

The $60^{\text {st }}$ and $80^{\text {th }}$ images

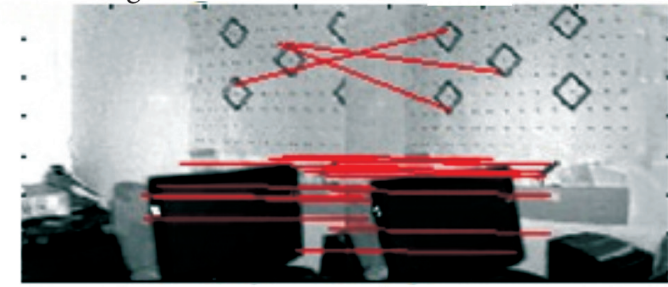

The $80^{\text {st }}$ and $100^{\text {th }}$ images

Fig. 5. The keypoints were extracted from the amplitude images by using SIFT. Three outlier keypoints between 80 th and 100th images were removed 
Table 2. The registration results achieved by SIFT and residuals on the keypoints (case study 3 )

\begin{tabular}{|c|c|c|c|c|c|}
\hline $\begin{array}{c}\text { Themeasurements } \\
\text { (frames) }\end{array}$ & $\begin{array}{l}\text { Matched } \\
\text { keypoints }\end{array}$ & $\begin{array}{c}\text { Rotations (grad) } \\
\text { Omega } \\
\text { Phi } \\
\text { Kappa }\end{array}$ & $\begin{array}{c}\text { Translations }(\mathrm{m}) \\
t x \\
t y \\
t z\end{array}$ & $\begin{array}{l}\text { Mean residual on } \\
\text { the keypoints }(\mathrm{m}) \\
\qquad \begin{array}{c}d x \\
d y \\
d z\end{array}\end{array}$ & $\begin{array}{c}\text { Std. dev. of keypoint } \\
\text { residuals }(\mathrm{m}) \\
s x \\
s y \\
s z\end{array}$ \\
\hline $1^{\text {th }}<20^{\text {th }}$ & 11 & $\begin{array}{l}4.71140 \\
0.74704 \\
0.78677 \\
\end{array}$ & $\begin{array}{l}-0.38778 \\
-0.17630 \\
-0.10048 \\
\end{array}$ & $\begin{array}{c}-1.9385 \mathrm{e}-009 \\
-5.7704 \mathrm{e}-010 \\
8.8261 \mathrm{e}-012 \\
\end{array}$ & $\begin{array}{l}0.02314 \\
0.01402 \\
0.05214 \\
\end{array}$ \\
\hline $20^{\text {th }} \leftarrow 40^{\text {th }}$ & 5 & $\begin{array}{c}3.71000 \\
18.19000 \\
-6.84000 \\
\end{array}$ & $\begin{array}{l}0.89760 \\
0.27585 \\
0.26410\end{array}$ & $\begin{array}{c}-8.1036 \mathrm{e}-006 \\
7.9602 \mathrm{e}-006 \\
-2.3161 \mathrm{e}-011\end{array}$ & $\begin{array}{l}0.04377 \\
0.05132 \\
0.01840\end{array}$ \\
\hline $40^{\text {th }} \leftarrow 60^{\text {th }}$ & 24 & $\begin{array}{c}2.48490 \\
18.28400 \\
-5.02480 \\
\end{array}$ & $\begin{array}{c}-0.33943 \\
-0.08431 \\
0.10434 \\
\end{array}$ & $\begin{array}{c}-1.8379 \mathrm{e}-006 \\
-1.6576 \mathrm{e}-008 \\
6.7925 \mathrm{e}-012 \\
\end{array}$ & $\begin{array}{l}0.04443 \\
0.02330 \\
0.10132\end{array}$ \\
\hline $60^{\text {th }}<80^{\text {th }}$ & 20 & $\begin{array}{l}-1.06430 \\
14.89200 \\
-2.47530 \\
\end{array}$ & $\begin{array}{c}-0.10970 \\
0.05749 \\
0.05107\end{array}$ & $\begin{array}{c}-5.2778 \mathrm{e}-007 \\
6.7366 \mathrm{e}-010 \\
1.1238 \mathrm{e}-011 \\
\end{array}$ & $\begin{array}{l}0.01809 \\
0.01883 \\
0.08151\end{array}$ \\
\hline $80^{\text {th }} \leftarrow 100^{\text {th }}$ & 16 & $\begin{array}{r}-1.00720 \\
16.14200 \\
-1.06740 \\
\end{array}$ & $\begin{array}{c}-0.00120 \\
0.04780 \\
0.00640\end{array}$ & $\begin{array}{c}-1.6514 \mathrm{e}-007 \\
-1.7038 \mathrm{e}-010 \\
1.6585 \mathrm{e}-013\end{array}$ & $\begin{array}{l}0.01048 \\
0.00954 \\
0.03945\end{array}$ \\
\hline
\end{tabular}

Table 3. The ICP results that were performed to SIFT results

\begin{tabular}{|c|c|c|}
\hline The frames & $\begin{array}{c}\text { Mean } \\
\text { convergence }(\mathrm{m})\end{array}$ & Std. dev. (m) \\
\hline $1^{\text {st }}<20^{\text {th }}$ & 0.000561 & 0.018563 \\
\hline $20^{\text {th }}<40^{\text {th }}$ & -0.000169 & 0.019318 \\
\hline $40^{\text {th }}<60^{\text {th }}$ & -0.000518 & 0.018512 \\
\hline $60^{\text {th }}<80^{\text {th }}$ & 0.001130 & 0.014539 \\
\hline $80^{\text {th }}<100^{\text {th }}$ & 0.000712 & 0.012499 \\
\hline
\end{tabular}

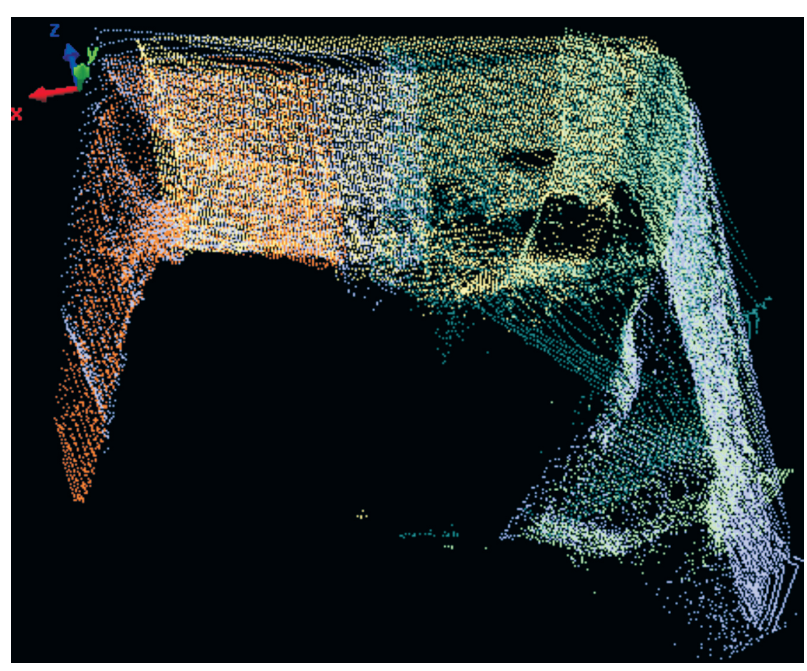

Fig. 6. The registered 3D point clouds of the measurement side

The coordinates of the keypoints were computed from their point clouds. The first point cloud was selected as reference, and all the others were successively registered in relation to its coordinate system. The registration parameters (Table 2) were computed with coordinates of the keypoints using Helmert 3D registration (1).

$$
\left[\begin{array}{l}
X \\
Y \\
Z
\end{array}\right]_{i}=\lambda \cdot R_{\omega \phi \chi} \cdot\left[\begin{array}{c}
X \\
Y \\
Z
\end{array}\right]_{j}+\left[\begin{array}{c}
t x \\
t y \\
t z
\end{array}\right], i=\text { reference and } i<j
$$

The accuracy of both the keypoint coordinates and the registration parameters are related to the pixel size the other word distance between the measured points. The current results of the registrations (Table 2) can be improved by the ICP. In that case, The registration was also performed by the ICP and more precise results were obtained (Table 3) (Fig. 6). Nevertheless, a large number of point clouds on the mobile applications by the RIM camera should not be registered consecutively to prevent cumulative errors. Hence, the measurement loops must be constituted or the first and the last measurements have to be consisted of control points belong to same coordinate system.

\section{Conclusions}

The laser scanners are fast and precise measurement techniques in comparison to conventional and photogrammetric methods. However they are not as widespread as expected because of their costs. On the other hand, due to its ground based property, it can't visualize all details. The results of this study show that the RIM camera can be used for 3D modelling tasks of indoor environment and objects. Its measurement accuracy is enough for $3 \mathrm{D}$ modelling studies, and their costs are very low with respect to the laser scanner. However, the short range and colorless measurements are deficiency of them. The object details 
can't be selected from the measurements because of their colorless point clouds. The color record for the points can be overcome by using the image of exterior camera. The mesh and texture mapped 3D model can also be constituted from point clouds of the camera. Furthermore the ambient light affects their performance. The camera should not be used in direct sunlight. The RIM camera is practical instrument for 3D measurement and modelling of indoor environments. It will be used for plenty of applications depending on the improvement of its technical specifications by the time.

In this study, the point clouds of static measurements were registered by the ICP with high accuracy. In addition, the registration of hand-held measurements were performed with the keypoints extracted by the SIFT from the amplitude images. Furthermore the keypoint based registrations were improved by the ICP method.

\section{Acknowledgements}

This study was funded by The Scientific and Technological Research Council of Turkey (TUBITAK) with project number $112 \mathrm{Y} 025$.

\section{References}

Altuntas, C. 2011. An experimental study on registration threedimensional range images using range and intensity data, International Archives of the Photogrammetry, Remote Sensing and Spatial Information Sciences 38(3/W22): 115-118.

Bay, H.; Ess, A.; Tuytelaars, T.; Gool, L. V. 2008. SURF: speeded up robust features, Computer Vision and Image Understanding 110(3): 346-359. http://dx.doi.org/10.1016/j.cviu.2007.09.014

Besl, P. J.; McKay, N. D. 1992. A method for registration of 3-D shapes, IEEE Transactions on Pattern Analysis and Machine Intelligence 14(2): 239-256.

http://dx.doi.org/10.1109/34.121791

Boehm, J.; Pattinson, T. 2010. Accuracy of exterior orientation for a range camera, International Archives of the Photogrammetry, Remote Sensing and Spatial Information Sciences 38(5): 103-108.

Brazetti, L.; Scaioni, M.; Remondino, F. 2010. Orientation and 3D modelling from markerlessterrestrial images: combining accuracy with automated, The Photogrammetric Record 25(132): 356-381.

http://dx.doi.org/10.1111/j.1477-9730.2010.00599.x

Centeno, J.; Jutzi, B. 2010. Evaluation of a range imaging sensor concerning resolution and illumination, International Archives of the Photogrammetry, Remote Sensing and Spatial Information Sciences 38(1): on CD.

Chen, Y.; Medioni, G. 1992. Objectmodelling by registration of multiple range images, Image and Vision Computing 10(3): 145-155. http://dx.doi.org/10.1016/0262-8856(92)90066-C

Cui, Y.; Schuon, S.; Chan, D.; Thrun, S.; Theobalt, C. 2010. $3 \mathrm{D}$ shape scanning with a time-of-flight camera, in IEEE
Conference on Computer Vision and Pattern Recognition, 13-18 June, 2010, San Francisco, CA, 1173-1180.

Fischler, M. A.; Bolles, R. C. 1981. Random sample consensus: a paradigm for model fitting with applications to image analysis and automated cartography, Communications Association and Computing Machine 24(6): 381-395.

http://dx.doi.org/10.1145/358669.358692

Frank, B.; Schmedding, R.; Stachniss, C.; Teschner, M.; Burgard, W. 2010. Learning deformable object models for mobile robot path planning using depth cameras and a manipulation robot, in Proceeding of the Workshop on Advanced Reasoning with Depth Cameras at Robotics: Science and Systems Conference (RSS), 27 June, 2010, Zaragoza, Spain. 6 p.

Hussmann, S.; Ringbeck, T.; Hagebeuker, B. 2008. A performance review of 3D TOF vision systems in comparison to stereo vision systems, in A. Bhatti (Ed.). Stereo Vision [online], [cited 23 February 2013]. ISBN: 978-953-7619-22-0. Available from Internet: http://www.intechopen.com/books/ stereo_vision/a_performance_review_of_3d_tof_vision_ systems_in_comparison_to_stereo_vision_systems

InnovMetric. 2007. PolyWorks Software, Beginnfer's Guide [online], [cited January 2013]. Available from Internet: http:// www.innovmetric.com/polyworks/3D-scanners/home. aspx?lang=en

Kahlmann, T.; Remondino, F.; Ingensand, H. 2006. Calibration for increased accuracy of the range imaging camera SwissRanger ${ }^{\mathrm{TM}}$, International Archives of the Photogrammetry, Remote Sensing and Spatial Information Sciences 36(5): 136-141.

Lichti, D. D.; Kim, C.; Jamtsho, S. 2010. An integrated bundle adjustment approach to range camera geometric self-calibration, ISPRS Journal of Photogrammetry and Remote Sensing 65(2010): 360-368. http://dx.doi.org/10.1016/j.isprsjprs.2010.04.002

Lowe, D. 2005. Demo software: SIFT keypointdetector (version 4, July 2005) [online], [cited 20 April 2011]. Available from Internet: http://www.cs.ubc.ca/ lowe/keypoints/.

Lowe, D. 2004. Distinctive image features from scale-invariant keypoints, International Journal of Computer Vision 60(2): 91110. http://dx.doi.org/10.1023/B:VISI.0000029664.99615.94

MesaImaging. 2012. SwissRanger SR4000 overview [online], [cited 10 October 2012]. Available from Internet: http://www. mesa-imaging.ch/prodview4k.php

Oggier, T.; Büttgen, B.; Lustenberger, F.; Becker, G.; Rüegg, B.; Hodac, A. 2005. SwissRanger SR3000 and first experiences based on miniaturized 3D-TOF cameras, in Proc. $1^{\text {st }}$ Range Imaging Research Day, 8-9 September, 2005, Zurich, Switzerland, 97-108.

Oggier, T.; Lehmann, M.; Kaufmann, R.; Schweizer, M.; Richter, M.; Metzler, P.; Lang, G.; Lustenberger, F.; Blanc, N. 2004. An all-solid-state optical range camera for 3D realtime imaging with sub-centimeterdepth resolution (SwissRanger), in Proceedings of the SPIE 5249: 534-545. http://dx.doi.org/10.1117/12.513307

Piatti, D. 2010. Time-of-flight cameras: tests, calibration and multi frame registration for automatic $3 D$ object reconstruction. PhD Thesis, Politecnico di Torino Doctoral school of Environment and Territory, Italy.

Rapp, H. 2007. Experimental and theoretical investigation of correlating TOF-camera systems. Diploma Thesis in Physics, Faculty of Physics and Astronomy, University of Heidelberg, Germany. 
Sturm, J.; Konolige, K.; Stachniss, C.; Burgard, W. 2010. 3D pose estimation, tracking and model learning of articulated objects from dense depth video using projected texture stereo, in Proceeding of the Workshop on Advanced Reasoning with Depth Cameras at Robotics: Science and Systems Conference (RSS), 27 June, 2010, Zaragoza, Spain. $6 \mathrm{p}$.

Teizer, J. 2008. 3D range imaging camera sensing for active safety in construction, ITcon 13: 103-117.
Cihan ALTUNTAS. He has PhD degree in geomatics engineering, and researcher in Selcuk University currently. He also performed scientific research in Vienna University of Technology and Calgary University. His study of interest is photogrammetry, aerial and ground based laser scanning, computer vision, three-dimensional modelling, range imaging camera and data fusion of multi sensor. He has many published scientific papers and performed review processes in many qualifying journals.

Ferruh YILDIZ. He is professor and lecturer in geomatics engineering of Selcuk University. His study of interest is aerial and close range photogrammetry, three-dimensional modelling and computer sciences. He has many papers published in scientific journals. 